\title{
On the coexistence of innovators and imitators
}

\author{
Roy Cerqueti $^{a}$, Fabio Tramontana ${ }^{b}$, Marco Ventura $^{c *}$ \\ ${ }^{a}$ Department of Economics and Law, University of Macerata, Italy \\ ${ }^{b}$ Department of Economics and Management, University of Pavia \\ ${ }^{c}$ Italian National Institute of Statistics (ISTAT), Rome, Italy
}

February 18, 2014

\begin{abstract}
The article develops a model laying a foundation for the idea that the relationships between competitors in the knowledge diffusion market can be described by a LotkaVolterra system. The model can accommodate both the scenario of prey-predator and that of competition between innovators and imitators. Analytic results and numerical simulations show that a stable coexistence equilibrium is feasible under both scenarios. The work also discusses the conditions under which these results are achievable.
\end{abstract}

Keywords: imitation; innovation; Lotka-Volterra system; knowledge diffusion; intellectual property right.

JEL Classification: O31; O33; O34; C6

\section{Introduction}

The controversial issue of protection of intellectual property rights (IPR), or more generally innovation, has been long debated and actually it does not seem to come at an end. The major reason of controversy emerges because of the presence of a trade-off faced by authorities when deciding the IPR degree of protection. In turn, the trade-off emerges from the public good nature of knowledge. At one extreme of the trade-off there are the alleged benefits that IPR convey to society. In particular, IPR increase the incentives to invest resources in the creation of new technological knowledge because of the positive effects in terms of appropriability and tradability of the new knowledge. For this reason, in recent years, many countries have put into place more effective or rigorous protection policies, such as the establishment of the Court of Appeals of the Federal Circuit by US Congress and the EU directive 2004/48 on the enforcement of intellectual property rights. At the other extreme, strong protection brings about drawbacks by creating monopolies.

One of the way followed in the literature to model the degree of IPR protection is to introduce imitation, via an exogenous imitation rate, competing with innovation, as imitation is commonly considered as an inverse measure of IPR protection (Helpman 1993, Lai 1998, Cysne and Turchick 2012). Among many, Furukawa (2007) finds that under some

\footnotetext{
${ }^{*}$ Corresponding author. Italian National Institute of Statistics (ISTAT), Econometric Studies and Economic Forecasting Division, p.zza Dell'Indipendenza4, -00185, Rome, Italy. Tel.:+36(0)6 4673 3665. Fax: +39(0)6 233232 419. Email: mventura@istat.it
} 
circumstances, the rate of innovation has an inverse-U shape as a function of imitation. This theoretical finding is also supported by Aghion et al. (2005) who find strong evidence of an inverted-U relationships between competition and innovation. At its very essence this strand of the literature implies that relaxing IPR to a certain extent, i.e. allowing for imitation, can be beneficial.

In the literature related to the broad field of knowledge diffusion, the Lotka-Volterra system has been extensively used (Bharagava 1989; Morris and Pratt 2003; Watanabe et al. 2003; Castiaux 2007; Lee et al. 2005; Kim et al 2006, just to cite a few). However, all of these contributions assume a Lotka-Volterra type joint dynamics, none of them derive it. Differently, our contribution aims at moving a first step to start filling this gap. In agreement with the aforementioned literature we start out from the idea that imitation plays an important role in speeding up the rate of innovation diffusion and is an inverse measure of IPR protection, in other words imitation can accidentally mitigate the innovation diffusion lag. ${ }^{1}$ In the model presented in this paper, innovators and imitators are regarded as competing for the same asset and entry the market requires undergoing sunk costs. Expanding the methodology proposed by Dixit and Pindyck (1994) we show that it is possible to derive the long run joint dynamics of imitators and innovators as a LotkaVolterra system. To this extent, our theoretical contribution provides theoretical support to the evolutionary view of knowledge diffusion.

More precisely, we derive the joint dynamics of imitators and innovators under two scenarios which are obtained through an appropriate selection of the variation range of the parameters of the system. The two scenarios are consistent with prey-predator interactions, in which innovators are regarded as preys and imitators as predators, and competitive interactions, occurring when both species suffer from each other's existence.

Analytical results and numerical simulations show that among the three possible types of equilibria (extinction, one-category and coexistence) the coexistence equilibrium -i.e.: the equilibrium associated to the simultaneous existence of innovators and imitators in the long run - can be achieved under both scenarios and it is a stable configuration. From an economic point of view, the circumstances under which this result is achievable can be read in the sense that in order to coexist, the relationship between the two species must be thoroughly balanced. A certain amount of competition between the two sub-populations is desirable, but there must be a limit to the extent to which one population can hamper the others' activity. This result is in line with the above cited literature claiming that stronger IPR protection is not always the best possible choice.

The article proceeds as follows. Section 2 presents the set up of the model. The derivation of innovators and imitators entry rates follow in Section 3 and the solutions of the system is presented in Section 4. Section 5 offers an economic interpretation of the results of the previous Section. Finally, Section 6 concludes.

\section{The set up of the model}

Consider an industry composed of a given number of firms. Each firm is risk neutral, acts competitively and has rational expectations about the underlying stochastic process and the decision rules of other firms. Moreover, each firm has the capacity to produce the flow

\footnotetext{
${ }^{1}$ Caballero and Jaffe (1993) estimate that the median lag between a cited patent and the citing patent is 9-10 years.
} 
of one unit of output, which it can activate by incurring a sunk cost. There are no variable costs of production and the elasticity of demand is large enough to ensure that each firm that has paid its sunk cost will want to produce at its capacity level. Uncertainty is firmspecific or independent across firms and the inverse demand function for each firm is of the type:

$$
P_{t}=Y_{t} D\left(Q_{t}\right), \quad t>0,
$$

where $P_{t}$ is the price faced by the firm at time $t, Q_{t}$ is the current output flow at time $t$, $D\left(Q_{t}\right)$ is a decreasing function, comprising the non-stochastic part of the inverse demand function. As each firm produces one unit of output, the current output flow equals the number of active firms, which, consistently with Dixit and Pindyck (1994 p. 251), we treat as a continuous variable. ${ }^{2} Y_{t}$ can be interpreted as an idiosyncratic demand shock reflecting changes in relative tastes for the firms' products, ultimately capturing a shift to profitability at time $t$. As in Dixit and Pindyck (1994, pp. 250 and 267), these shocks can be the source of a competitive advantage that allows firms to enter the industry acting either as innovators or as imitators. By paying an entry cost $R$, any firm can get an initial draw $Y_{0}$ of its demand shock $Y_{t}$ from a known distribution. Thereafter $\left\{Y_{t}\right\}_{t>0}$ will follow a geometric Brownian motion process that is firm-specific, or independent across firms:

$$
d Y_{t}=\alpha Y_{t} d t+\sigma Y_{t} d z_{t} \quad t>0
$$

where $\left\{z_{t}\right\}_{t>0}$ is a standard Brownian motion while $\alpha \in \mathbb{R}$ and $\sigma>0$ represent the drift and the diffusion coefficients of the stochastic process $\left\{Y_{t}\right\}_{t>0}$, respectively.

After the payment of the cost $R$, a firm observes the value $Y_{0}$. Each firm can start actual operation by paying a further sunk cost. Thus, some firms decide to invest in the development of new products, and hence act as innovators, while other firms aim at reproducing the innovations performed by the innovators, being so imitators.

If $Y_{0}$ exceeds a critical threshold $Y^{(N)}$, a would-be innovator pays the investment cost $I$ and becomes an active producer. Otherwise, it lets $\left\{Y_{t}\right\}_{t>0}$ evolve and activates if and when $Y^{(N)}$ is reached. Analogously, a would-be imitator pays a fixed investment cost $K$, with $K<I$, to enter the market and appropriate a share of the innovators' income if and when $\left\{Y_{t}\right\}_{t>0}$ randomly fluctuating exceeds a critical threshold $Y^{(M)}$. Otherwise, it keeps waiting and lets $\left\{Y_{t}\right\}_{t>0}$ evolve. Let us denote as $N_{t}, M_{t}$ respectively, the number of innovators and imitators at time $t$ that will reach the activation decision. We assume in our model that the activation thresholds $Y^{(N)}$ and $Y^{(M)}$ vary with the number of innovators and imitators which are currently active in the market: substantially, at time $t$ the activation thresholds are $Y^{(N)}=Y^{(N)}\left(N_{t}, M_{t}\right)$ and $Y^{(M)}=Y^{(M)}\left(N_{t}, M_{t}\right)$.

We clarify the stylized points set out so far by means of a simple example.

Example 1. $R$ can be representative of a situation where a pharmaceutical company can develop a new drug by incurring the research cost. The would-be innovator patents the drug, but unless the profit estimate is sufficiently high, i.e. whenever it reaches a threshold, the firm will not incur the additional investment expenditure $I$ that is necessary to begin production. Such a profit threshold is affected by the number of firms currently working on a very similar project, either as potential innovators or as potential imitators.

\footnotetext{
${ }^{2} \mathrm{~A}$ formal rigorous treatment of the resulting continuum of random variables and their law of large numbers would be far too lengthy and out of the scope of this paper. For the basis of rigorous theory we refer to Judd (1985).
} 


\section{Derivation of the entry rates}

This section derives the rates at which innovators and imitators activate. We adopt and extend to our case the methodology proposed by Dixit and Pindyck (1994).

First of all, we state a balance flow assumption, i.e.: the number of active firms is constant over time, namely in each instant of time the number of firms reaching the activation decision is equal to the number of firms that become idle. In a long run equilibrium this assumption is made possible thanks to the fact that uncertainty is firm-specific, namely firms' shocks are independent, and the operating of the law of large numbers ensures that industry aggregates are non-random. Hence, a non-random total volume of output can be produced by firms whose identities change through time but whose aggregate population distribution remains stationary.

To formalize this condition, we introduce a Poisson death process ${ }^{3}$ at rate $\lambda$.

We will now deal with innovators' activation rate. Let us define the process $\left\{w_{t}\right\}_{t>0}=$ $\left\{\log \left(Y_{t}\right)\right\}_{t>0}$ and the threshold $w^{(N)}=\log \left(Y^{(N)}\right)$, where $\left\{Y_{t}\right\}_{t>0}$ is a geometric Brownian motion as in (2). Applying Ito's lemma it can be easily verified that the dynamics of $\left\{w_{t}\right\}_{t>0}$ follows a Brownian motion of the type

$$
d w_{t}=\theta d t+\sigma d z, \quad t>0
$$

with $\theta=\alpha-\frac{\sigma^{2}}{2}$. Let $g(w)$ be the density function of the initial value $w_{0}$ of $\left\{w_{t}\right\}_{t \geq 0}$ and $G(w)$ the corresponding distribution function, with $w \in \mathbb{R}$.

The agents who are candidates for the innovation are distributed continuously between the (log of the) minimum value of the expected gain -i.e.: $\log \left(0^{+}\right)=-\infty$ - and the (log of the) innovation threshold value -i.e.: $\log \left(Y^{(N)}\right)=w^{(N)}$.

We define the instantaneous rate at time $t$ at which innovators activate as $N_{t}^{\prime}=\frac{d N_{t}}{d t}$, being $d N_{t}$ and $d t$ small variations of $N_{t}$ and $t$, respectively. Now, let us introduce the function $\phi:\left(-\infty, w^{(N)}\right) \rightarrow(0,1)$ such that $N_{t}^{\prime} \phi(w)$ is the density of these agents at location $w$. For notational simplicity we will omit the index $t$ hereafter.

For the density to be stable over time (under the balance flow assumption), we require the agents leaving the location at $w$ (having received shocks of the Brownian motion process or Poisson death) to be exactly counterbalanced by the agents arriving in the location (having received positive shocks from below or negative shocks from above). In order to formalize this condition, we denote as $d h=\sigma \sqrt{d t}$ a small variation in the net gain $w$. Some of the firms located in $d h$ will die by a proportion $\lambda d t$. Among the survivors, a fraction $p$ will move to the right, i.e. will activate, and a fraction $1-p$ will move to the left, i.e. will exit the market. The parameter $p$ can be found using the binomial approximation of the Brownian motion, namely $p=\frac{1}{2}\left(1+\frac{\theta \sqrt{d t}}{\sigma}\right)$. Therefore, the stability condition can be written as follows:

$$
N^{\prime} \phi(w) d h=N^{\prime} d t g(w) d h+p(1-\lambda d t) N^{\prime} \phi(w-d h) d h+(1-p)(1-\lambda d t) N^{\prime} \phi(w+d h) d h .
$$

By deleting the common factor $N^{\prime} d h$, equation (3) becomes:

$$
\phi(w)=g(w) d t+p(1-\lambda d t) \phi(w-d h)+(1-p)(1-\lambda d t) \phi(w+d h) .
$$

\footnotetext{
${ }^{3}$ By death or mortality rate it is meant the rate at which firms exit the market.
} 
A Taylor expansion gives:

$$
\left\{\begin{array}{l}
\left.\phi(w-d h)\right|_{d h=0}=\phi(w)-\phi^{\prime}(w) d h+\phi^{\prime \prime}(w) \frac{d h^{2}}{2} \\
\left.\phi(w+d h)\right|_{d h=0}=\phi(w)+\phi^{\prime}(w) d h+\phi^{\prime \prime}(w) \frac{d h^{2}}{2}
\end{array}\right.
$$

By substituting (5) and the approximation $p=\frac{1}{2}\left(1+\frac{\theta \sqrt{d t}}{\sigma}\right)$ into equation (3) we obtain:

$$
g(w)-\lambda \phi(w-d h)-\theta(1-\lambda d t) \phi^{\prime}(w)+(1-\lambda d t) \phi^{\prime \prime}(w) \cdot \frac{\sigma^{2}}{2}=0 .
$$

Taking the limit for $d t \rightarrow 0$, expression (6) becomes a differential equation with constant coefficients and variable term:

$$
\frac{\sigma^{2}}{2} \phi^{\prime \prime}(w)-\theta \phi^{\prime}(w)-\lambda \phi(w)+g(w)=0
$$

The general solution of equation (7) is of the type:

$$
\phi(w)=C_{1} \exp \left(b_{1} w\right)+C_{2} \exp \left(b_{2} w\right)+\phi_{0}(w)
$$

where $C_{1}$ and $C_{2}$ will be pinned down, as we will see shortly, through the fulfilment of some boundary conditions and $b_{1}$ and $b_{2}$ are the roots of the characteristic equation

$$
\frac{\sigma^{2}}{2} b^{2}-\theta b-\lambda=0
$$

i.e.:

$$
b_{1}=\frac{\theta+\sqrt{\theta^{2}+2 \lambda \sigma^{2}}}{\sigma^{2}}>0, \quad b_{2}=\frac{\theta-\sqrt{\theta^{2}+2 \lambda \sigma^{2}}}{\sigma^{2}}<0
$$

while $\phi_{0}$ is a particular solution to the differential equation in (7), and it vanishes as $w \rightarrow-\infty$.

For analytical tractability, let us assume now that the initial payoff, $Y_{0}$, is distributed uniformly, with bounded support over the range $\left(0, Y^{*}\right)$. Analogously, the logarithm of the initial payoff, $w_{0}$, is distributed according to an exponential function: $g(w)=\exp \left(w-w^{*}\right)=$ $G(w)$, with $w^{*}=\log \left(Y^{*}\right)$, meaning that the initial draw of $\left\{w_{t}\right\}_{t>0}$ is taken from an exponential random variable. In this case, it can be easily verified that a specific solution $\phi_{0}$ is given by:

$$
\phi_{0}(w)=\frac{\exp \left(w-w^{*}\right)}{\lambda+\theta-\frac{\sigma^{2}}{2}}
$$

with $\frac{\sigma^{2}}{2}<\lambda+\theta$ to make economic sense. This condition implies the positive root of the quadratic expression to be greater than unity, $b_{1}>1$. The definition of $\phi$ provides us with the boundary conditions, that are:

$$
\left\{\begin{array}{l}
\lim _{w \rightarrow-\infty} \phi(w)=0 \\
\lim _{w \rightarrow w^{(N)-}} \phi(w)=0
\end{array}\right.
$$


The second line in (11) is due to the fact that the mass of waiting firms is zero at the entry threshold and it comes out from a simple computation (see Dixit and Pyndyck, 1994), while the first line is a simple restatement of the fact that a probability distribution function takes value zero at its lower limit. By exploiting the boundary conditions in (11) we can determine the two constants as:

$$
\left\{\begin{array}{l}
C_{2}=0 ; \\
C_{1}=-\frac{\exp \left[w^{(N)}\left(1-b_{1}\right)-w^{*}\right]}{\left(\lambda+\theta-\frac{\sigma^{2}}{2}\right)} .
\end{array}\right.
$$

By substituting (10) and (12) into (8) we finally obtain the solution of the differential equation, i.e.:

$$
\phi(w)=-\frac{\exp \left[w^{(N)}\left(1-b_{1}\right)-w^{*}+b_{1} w\right]-\exp \left(w-w^{*}\right)}{\left(\lambda+\theta-\frac{\sigma^{2}}{2}\right)} .
$$

The innovators' activation rate is the rate at which waiting firms hit $w^{(N)}$. This is given by the fraction $p(1-\lambda d t)$ of the firms located just at the left of $w^{(N)}$.

Using a Taylor expansion on $\phi\left(w^{(N)}-d h\right)$ for $d h=0$, the approximation $p=\frac{1}{2}\left(1+\frac{\theta \sqrt{d t}}{\sigma}\right)$ and by taking the limit for $d t \rightarrow 0$, then (10), (11) and (13) give:

$$
\begin{gathered}
\lim _{w \rightarrow w^{(N)}-} \frac{1}{2} N^{\prime}\left[\phi(w)-\phi^{\prime}(w) d h\right] d h=-\lim _{w \rightarrow w^{(N)}-} \frac{1}{2} N^{\prime} \phi^{\prime}(w)(d h)^{2}= \\
=-\lim _{w \rightarrow w^{(N)}-} \frac{\sigma^{2}}{2} N^{\prime} \phi^{\prime}(w) d t=N^{\prime} \frac{\frac{\sigma^{2}}{2}\left(b_{1}-1\right)}{\left(\lambda+\theta-\frac{\sigma^{2}}{2}\right)} \exp \left(w^{(N)}-w^{*}\right),
\end{gathered}
$$

being the last term equal to the rate of activation, since these firms activate in the time interval $d t$.

We now move on to the case of imitators. The procedure is analogous to that just described for innovators, and consistently, we adopt a very similar notation.

We firstly define the threshold $w^{(M)}=\log \left(Y^{(M)}\right)$. The agents who are candidates for imitating activity are distributed continuously in $\left(-\infty, w^{(M)}\right)$. We define the instantaneous rate at time $t$ at which imitators activate as $M_{t}^{\prime}=\frac{d M_{t}}{d t}$, being $d M_{t}$ and $d t$ small variations of $M_{t}$ and $t$, respectively. We also introduce the function $\psi:\left(-\infty, w^{(M)}\right) \rightarrow(0,1)$ such that $M_{t}^{\prime} \psi(w)$ is the density of imitators at location $w$.

The balance flow assumption leads to:

$M^{\prime} \psi(w) d h=M^{\prime} d t g(w) d h+p(1-\lambda d t) M^{\prime} \psi(w-d h) d h+(1-p)(1-\lambda d t) M^{\prime} \psi(w+d h) d h$,

which becomes the following differential equation:

$$
\frac{\sigma^{2}}{2} \psi^{\prime \prime}(w)-\theta \psi^{\prime}(w)-\lambda \psi(w)+g(w)=0 .
$$

By assuming that $Y_{0}$ follows a uniform distribution over $\left(0, Y^{*}\right)$, we obtain the general solution of equation (16) as:

$$
\psi(w)=-\frac{\exp \left[w^{(M)}\left(1-b_{1}\right)-w^{*}+b_{1} w\right]-\exp \left(w-w^{*}\right)}{\left(\lambda+\theta-\frac{\sigma^{2}}{2}\right)} .
$$


where $\psi$ satisfies the boundary conditions:

$$
\left\{\begin{array}{c}
\lim _{w \rightarrow-\infty} \psi(w)=0 \\
\lim _{w \rightarrow w^{(M)-}} \psi(w)=0
\end{array}\right.
$$

The rate of activation of the imitators is the one at which the waiting firms hit $w^{(M)}$, and it is given by:

$$
\begin{gathered}
\lim _{w \rightarrow w^{(M)}-} \frac{1}{2} M^{\prime}\left[\psi(w)-\psi^{\prime}(w) d h\right] d h=-\lim _{w \rightarrow w^{(M)}-} \frac{1}{2} M^{\prime} \psi^{\prime}(w)(d h)^{2}= \\
=-\lim _{w \rightarrow w^{(M)}-} \frac{\sigma^{2}}{2} M^{\prime} \psi^{\prime}(w) d t=M^{\prime} \frac{\frac{\sigma^{2}}{2}\left(b_{1}-1\right)}{\left(\lambda+\theta-\frac{\sigma^{2}}{2}\right)} \exp \left(w^{(M)}-w^{*}\right) .
\end{gathered}
$$

We recall that the balance flow assumption states that the number of activating firms equals the number of firms exiting the market. This condition can be split into two assumptions, one for innovators and the other for imitators. Let us first consider the case of innovators. The number of activating firms is given by the sum of those firms who find their $w_{0}$ greater than the entry threshold plus the activation flow found in (14). Therefore, we must have:

$$
\begin{aligned}
& \lambda N=\lim _{w \rightarrow w^{(N)-}} N^{\prime}\left[1-G(w)-\frac{\sigma^{2}}{2} \phi^{\prime}(w)\right]= \\
& =N^{\prime}\left[1-\exp \left(w^{(N)}-w^{*}\right) \frac{\lambda+\theta-\frac{\sigma^{2}}{2} b_{1}}{\left(\lambda+\theta-\frac{\sigma^{2}}{2}\right)}\right] .
\end{aligned}
$$

Analogously, for imitators we can write:

$$
\begin{aligned}
& \lambda M=\lim _{w \rightarrow w^{(M)-}} M^{\prime}\left[1-G(w)-\frac{\sigma^{2}}{2} \psi^{\prime}(w)\right]= \\
& =M^{\prime}\left[1-\exp \left(w^{(M)}-w^{*}\right) \frac{\lambda+\theta-\frac{\sigma^{2}}{2} b_{1}}{\left(\lambda+\theta-\frac{\sigma^{2}}{2}\right)}\right] .
\end{aligned}
$$

Remark 1. Notice that the joint fulfilment of (20) and (21) provides only a sufficient condition for the balance flow assumption to be satisfied. However, this joint hypothesis is reasonable, not too restrictive and makes the model mathematically tractable.

For notational convenience, we define $\dot{n} \equiv \frac{N^{\prime}}{N}$ and $\dot{m} \equiv \frac{M^{\prime}}{M}$. Solving for $\dot{n}$ yields:

$$
\dot{n}=\frac{\lambda\left(\lambda+\theta-\frac{\sigma^{2}}{2}\right)}{\left(\lambda+\theta-\frac{\sigma^{2}}{2}\right)-e^{\left(w^{(N)}-w^{*}\right)}\left(\lambda+\theta-\frac{\sigma^{2}}{2} b_{1}\right)}=\frac{\lambda \Gamma}{\Gamma-\frac{Y^{(N)}}{Y^{*}}}
$$

with

$$
\Gamma \equiv \frac{\left(\lambda+\theta-\frac{\sigma^{2}}{2}\right)}{\left(\lambda+\theta-\frac{\sigma^{2}}{2} b_{1}\right)}>1
$$


For $\dot{m}$ we obtain:

$$
\dot{m}=\frac{\lambda\left(\lambda+\theta-\frac{\sigma^{2}}{2}\right)}{\left(\lambda+\theta-\frac{\sigma^{2}}{2}\right)-\frac{Y^{(M)}}{Y^{*}}\left(\lambda+\theta-\frac{\sigma^{2}}{2} b_{1}\right)}=\frac{\lambda \Gamma}{\Gamma-\frac{Y^{(M)}}{Y^{*}}}
$$

From (22) and (23) it appears clearly that the rates of entry are non-linear positive functions of the thresholds $Y^{(N)}$ and $Y^{(M)}$, respectively of innovators and imitators, and $\frac{d \dot{n}}{d Y^{(N)}}>0$ and $\frac{d \dot{m}}{d Y^{(M)}}>0$.

Taking a first order Taylor expansion of $\dot{n}$ and $\dot{m}$ around an arbitrary point $\left(N_{0}, M_{0}\right)$ equations (22) and (23) can be rewritten as:

$$
\left\{\begin{array}{l}
\dot{n}=\left.\dot{n}\right|_{M=M_{0}, N=N_{0}}+\left.\frac{d \dot{n}}{d N}\right|_{M=M_{0}, N=N_{0}}\left(N-N_{0}\right)+\left.\frac{d \dot{n}}{d M}\right|_{M=M_{0}, N=N_{0}}\left(M-M_{0}\right) \\
\dot{m}=\left.\dot{m}\right|_{M=M_{0}, N=N_{0}}+\left.\frac{d \dot{m}}{d N}\right|_{M=M_{0}, N=N_{0}}\left(N-N_{0}\right)+\left.\frac{d \dot{m}}{d M}\right|_{M=M_{0}, N=N_{0}}\left(M-M_{0}\right) .
\end{array}\right.
$$

By applying the chain rule $\frac{d \dot{n}}{d N}=\frac{\partial \dot{n}}{\partial Y^{(N)}} \frac{\partial Y^{(N)}}{\partial N}, \frac{d \dot{n}}{d M}=\frac{\partial \dot{n}}{\partial Y^{(N)}} \frac{\partial Y^{(N)}}{\partial M}$ into the Taylor expansion for $\dot{n}$ in (24), we obtain:

$$
\begin{gathered}
\dot{n}=\dot{n}_{0}+\frac{\partial \dot{n}}{\partial Y^{(N)}}\left[\frac{\partial Y^{(N)}}{\partial N}\left(N-N_{0}\right)+\frac{\partial Y^{(N)}}{\partial M}\left(M-M_{0}\right)\right]= \\
=\dot{n}_{0}-\frac{\partial \dot{n}}{\partial Y^{(N)}}\left(\frac{\partial Y^{(N)}}{\partial N} N_{0}+\frac{\partial Y^{(N)}}{\partial M} M_{0}\right)+\frac{\partial \dot{n}}{\partial Y^{(N)}}\left(\frac{\partial Y^{(N)}}{\partial N} N+\frac{\partial Y^{(N)}}{\partial M} M\right),
\end{gathered}
$$

where $\left.\dot{n}_{0} \equiv \dot{n}\right|_{M=M_{0}, N=N_{0}}$ and recalling that both the partial and the total derivatives are evaluated at the expansion point.

By rearranging the terms, system (25) can be rewritten as:

$$
\left\{\begin{array}{l}
\frac{N^{\prime}}{N}=r-s N-f M \\
\frac{M^{\prime}}{M}=g+c N-e M
\end{array}\right.
$$

with $r \equiv \dot{n}_{0}-\left(\frac{d \dot{n}}{d N} N_{0}+\frac{d \dot{n}}{d M} M_{0}\right) ; s \equiv-\frac{d \dot{n}}{d N} ; f \equiv-\frac{d \dot{n}}{d M} ; e \equiv-\frac{d \dot{m}}{d M} ; c \equiv \frac{d \dot{m}}{d N}$ and $g \equiv$ $\dot{m}_{0}-\left(\frac{d \dot{m}}{d N} N_{0}+\frac{d \dot{m}}{d M} M_{0}\right)$.

It is immediate to notice that in (26) the rates are function of both the number of innovators and the number of imitators. Such a dependence shows how the evolution of one of the two populations is intrinsically connected to the dynamics of the other. Therefore, in studying the evolution of the two groups one must take into account how they interact.

At a closer look (26) resembles very much to the prey-predator model elaborated by Volterra (1926) and popularized by Lotka (1956). In the literature related to innovation, and more broadly to knowledge diffusion, the Lotka-Volterra system has been extensively used (Bharagava 1989; Morris and Pratt 2003; Watanabe et al. 2003; Castiaux 2007; Lee et al 2005; Kim et al. 2006, just to cite a few). However, all of these contributions start out by assuming a Lotka-Volterra codynamics, none of them derive it. Differently, our contribution fills this gap showing that a Lotka-Volterra system can be consistently obtained as the resulting codynamics of two types of agents operating in a competitive industry. To this extent, our theoretical contribution lends support to that part of the literature on knowledge diffusion. 
In the Lotka-Volterra system the type of interaction between innovators and imitators is determined by the signs of the coefficients in (26) (Lee et al. 2005 and Kim et al. 2006). In order to determine such signs we use economic rationale as a guidance. Indeed, we assume the rate of entry of innovators to depend negatively on the total number of firms of any type. Therefore, the following composite condition holds

$$
\left\{\begin{array}{l}
\frac{d \dot{n}}{d N} \leq 0 \\
\frac{d \dot{n}}{d M} \leq 0
\end{array}\right.
$$

Notice that the first condition in (27) rules out an implausible explosive growth rate of innovators. In addition, from (22) we know that $\frac{d \dot{n}}{d Y^{(N)}}>0$ implying $\frac{\partial Y^{(N)}}{\partial N} \leq 0$, and thus we have found that the innovators' entry threshold is negatively related to the total number of active innovators. Put another way: the lower the threshold, the smaller the number of firms waiting to enter. This is consistent with the fact that new entrants are willing to accept a lower perspective profit when competition is more severe. Whereas, the second condition, $\frac{d \dot{n}}{d M} \leq 0$, captures the fact that imitators are detrimental to innovators, recalling again that $\frac{d \dot{n}}{d Y^{(N)}}>0$ it follows that $\frac{\partial Y^{(N)}}{\partial M} \leq 0$.

By the same token, to avoid the imitators growth rate to go off $\frac{d \dot{m}}{d M} \leq 0$ is required, from (23) we know that $\frac{d \dot{m}}{d Y^{(M)}}>0$ implying $\frac{\partial Y^{(M)}}{\partial M} \leq 0$. As regard to the condition $\frac{d \dot{m}}{d N}$, in agreement with economic theory, two different instances are possible and we put forward two proposals referred to as scenarios.

The first one, highlights that imitators entry rate must be a positive function of the number of active innovators as imitators thrive imitating. Hence, we consistently assume that $\frac{d \dot{m}}{d N} \geq 0$ which implies $\frac{\partial Y^{(M)}}{\partial N} \geq 0$. This first scenario can be labeled as prey-predator scenario or prey-predator interactions.

In the second scenario, we take into account the cases in which innovators can somehow kill imitators. In such cases (26) describes the evolution of two competing species. This occurrence can be easily captured by the term $\frac{d \dot{m}}{d N} \leq 0$, which, in turn, implies that the signs of some parameters change: $g>0$ and $c<0$. This situation can be representative of economies in which innovators are endowed with effective private and/or public protection of IPR, or whenever innovations make imitations obsolete. These conditions are more likely to occur in advanced economies. In other words $-c$ can be thought of as representing the degree of IPR protection. Hence, the second scenario can be referred to as competitive interactions.

To sum up, the two scenarios can be classified according to the signs of some parameters in (26) as shown by Table 1. In particular, we can say that in general $r, s, f, e>0$, and the

Table 1: Type of interactions according to the signs of $c$ and $f$

\begin{tabular}{|l|l|l|l|l|}
\hline \multicolumn{2}{|c|}{ sign of } & Type of interaction & scenario & explanation \\
\hline$c$ & $f$ & & 1 & $\begin{array}{l}\text { occurs when one specie (innovators) cannot } \\
\text { effectively protect itself }\end{array}$ \\
\hline+ & $+/-$ & prey-predator & 1 & $\begin{array}{l}\text { occurs when both species suffer from each } \\
\text { other's existence }\end{array}$ \\
\hline- & + & competition & 2 & \\
\hline
\end{tabular}

distinction between the two scenarios is given by the signs of $c$ and $f .{ }^{4}$

\footnotetext{
${ }^{4}$ We deliberately omit the analysis of cases in which one group affects the other but not vice-versa, as
} 
At this point, the canonical Lotka-Volterra system in (26) can be read as stating that in each period innovators increase by a proportion of $r$ and, at the same time, die out by "natural death" by a quadratic proportion $-s N_{t}^{2}$. The quadratic term, $-s N_{t}^{2}$, captures both the high riskiness inherent innovation activity and elbowing, namely competition among members of the same species for a limited resource. ${ }^{5}$

The last term of the first equation of (26) quantifies the rate of death induced by the coexistence of preys and predators, $f$, namely it points out that imitators kill innovators by imitating, as a metaphor of the profit loss suffered by innovators because of imitators. The greater the number of preys the higher the possibility of hunting for predators, and the greater the number of predators the greater the number of victims. Symmetrically, the law of evolution of the imitators, namely the imitators' growth rate, is supposed to be driven by an exogenous natural birth rate, $g$, and a natural quadratic death rate, $-e M_{t}$. In the case of imitators, the quadratic term can be thought of as capturing the double cause of mortality induced by either market conditions (i.e. competition among imitators, profitability and appropriability of profit produced by innovators), or by the effectiveness of IP protection system punishing imitators, or by a combination of both. Finally, in the imitators' evolution law there appears an extra term, $c$, indicating that imitators may either thrive by haunting innovators (prey-predator type of interaction) or compete with them (competition type of interaction).

From a mathematical point of view, Leslie (1957) shows that the system (26) can be rewritten in a discrete version as:

$$
\left\{\begin{array}{l}
N(t+1)=\frac{\alpha_{1} N(t)}{1+\beta_{1} N(t)+\gamma_{1} M(t)} \\
M(t+1)=\frac{\alpha_{2} M(t)}{1+\beta_{2} M(t)+\gamma_{2} N(t)}
\end{array}\right.
$$

where

$$
\left\{\begin{array}{l}
r=\log \left(\alpha_{1}\right) ; \\
g=\log \left(\alpha_{2}\right) ; \\
s=\frac{\beta_{1} \log \left(\alpha_{1}\right)}{\alpha_{1}-1} ; \\
e=\frac{\beta_{2} \log \left(\alpha_{2}\right)}{\alpha_{2}-1} ; \\
f=\frac{\gamma_{1} \log \left(\alpha_{1}\right)}{\alpha_{1}-1} ; \\
c=-\frac{\gamma_{2} \log \left(\alpha_{2}\right)}{\alpha_{2}-1} .
\end{array}\right.
$$

The relationships linking the continuous and the discrete version of the system stated in (29), can be easily exploited to formalize the two scenarios in a new fashion as:

Scen-1 $\alpha_{1}>1, \alpha_{2}>0, \beta_{1}>0, \beta_{2}>0, \gamma_{1}>0, \gamma_{2}<0$

Scen-2 $\alpha_{1}>1, \alpha_{2}>1, \beta_{1}>0, \beta_{2}>0, \gamma_{1}>0, \gamma_{2}>0$.

In what follows we will apply the result by Leslie (1957) as it allows to perform a more exhaustive analysis of the dynamic system.

these cases are not suitable for the economic context we are analyzing. In particular, not taking into account the other party's action does not fulfill economic rationality. These cases, referred to as commensalism and amensalism by biologists (see for instance Odum 1971 or Williamson 1972), are obtained by setting $c=0$ or $f=0$ and originate a system where a group evolves following a classic logistic growth and it drives the other. For the same reason we are not interested here in the case of neutralism $(c, f=0)$ where both groups evolve independently.

${ }^{5}$ Evidence about the high mortality rate among innovators can also be found in Christenses (1997), Christensen and Raynor (2003). 


\section{The solutions of the system}

The dynamic system in (28) is characterized by three kinds of equilibria: the total extinction equilibrium $E_{0}(0,0)$, the one-category equilibria in which only one kind of agents survives, and eventually the coexistence equilibrium. The one-category equilibria are characterized by the following Cartesian coordinates in the $(N ; M)$ plane:

$$
E_{N}\left(\frac{\alpha_{1}-1}{\beta_{1}} ; 0\right) \quad ; \quad E_{M}\left(0 ; \frac{\alpha_{2}-1}{\beta_{2}}\right)
$$

where $E_{N}$ and $E_{M}$ represent the equilibrium in which innovators or imitators are the only survivors, respectively. In particular $E_{M}$ makes sense only for $\alpha_{2}>1$, that is in Scen-2.

Remark 2. Although difficult to understand at a first instance, the $E_{M}$ equilibrium can exist, provided that it has been reached after a period of coexistence, which is the case being analyzed. Think of a situation characterized by weak IPR protection, in which a great deal of innovators' profit is captured by imitators. After a certain time period, innovators will be driven out of the market, meaning that they no longer find profitable innovating and either exit the market, or even become imitators themselves. After the extinction of innovators the market will be populated only by imitators, who still carry on producing and selling imitations. This stylized situation depicts many actual ones. Briefly speaking, this is what happened in Mauritius, where big Western companies in the fashion industry left the country, moving their production elsewhere and the local firms, having acquired the necessary know how, produce and sell imitations of original brands. In some cases, Mauritian firms have kept the 'cliché' and produce imitations with perfectly imitated logos. Another example can be provided by China, as a unique great market populated only by imitators. Yet, to a smaller dimension, in many corner markets it is not possible to find original innovative products, only imitations are sold, as they are typically cheaper.

The coexistence equilibrium is given by:

$$
E^{*}=\left(N^{*} ; M^{*}\right)=\left(\frac{\gamma_{2}\left(1-\alpha_{1}\right)-\beta_{1}\left(1-\alpha_{2}\right)}{\beta_{1} \beta_{2}-\gamma_{1} \gamma_{2}} ; \frac{\gamma_{1}\left(1-\alpha_{2}\right)-\beta_{2}\left(1-\alpha_{1}\right)}{\beta_{1} \beta_{2}-\gamma_{1} \gamma_{2}}\right)
$$

and it is meaningful when the two coordinates are strictly positive.

It is interesting to note the relative number of the two firms in the coexistence equilibrium, i.e. which group is the most numerous. We have the following result:

$$
N^{*} \gtreqless M^{*} \Longleftrightarrow\left(1-\alpha_{1}\right)\left(\gamma_{2}+\beta_{2}\right) \gtreqless\left(1-\alpha_{2}\right)\left(\gamma_{1}+\beta_{1}\right)
$$

In order to analyze the dynamic properties of the system we must study its Jacobian matrix:

$$
J:\left[\begin{array}{cc}
\frac{\alpha_{1}\left(1+\gamma_{1} M\right)}{\left(1+\beta_{1} N+\gamma_{1} M\right)^{2}} & -\frac{\alpha_{1} \gamma_{1} N}{\left(1+\beta_{1} N+\gamma_{1} M\right)^{2}} \\
-\frac{\alpha_{2} \gamma_{2} M}{\left(1+\beta_{2} M+\gamma_{2} N\right)^{2}} & \frac{\alpha_{2}\left(1+\gamma_{2} N\right)}{\left(1+\beta_{2} M+\gamma_{2} N\right)^{2}}
\end{array}\right]
$$

calculated at the different equilibrium points. Thus, for the extinction equilibrium the Jacobian becomes:

$$
J\left(E_{0}\right):\left[\begin{array}{cc}
\alpha_{1} & 0 \\
0 & \alpha_{2}
\end{array}\right]
$$


the eigenvalues of which are $\lambda_{1}^{0}=\alpha_{1}$ and $\lambda_{2}^{0}=\alpha_{2}$. We have assumed that $\alpha_{1}>1$, so the extinction equilibrium is never locally stable in our model.

The local stability properties of the one-category equilibria and of the coexistence equilibrium have been fully analyzed by Leslie and Gower (1958). ${ }^{6}$ In the following Proposition we adapt their results to our parameters.

Proposition 1. (Leslie and Gower, 1958) System (28) admits four possible cases:

I. if $\frac{\gamma_{1} \gamma_{2}}{\beta_{1} \beta_{2}}<\frac{\gamma_{1}\left(\alpha_{2}-1\right)}{\beta_{2}\left(\alpha_{1}-1\right)}<1$ then the coexistence equilibrium $E^{*}$ is stable;

II. if $1<\frac{\gamma_{1}\left(\alpha_{2}-1\right)}{\beta_{2}\left(\alpha_{1}-1\right)}<\frac{\gamma_{1} \gamma_{2}}{\beta_{1} \beta_{2}}$ then the coexistence equilibrium $E^{*}$ is unstable, while the two one-category equilibria are both locally stable and the system converges to one of them, depending on the initial levels of innovators and imitators;

III. if $\frac{\gamma_{1}\left(\alpha_{2}-1\right)}{\beta_{2}\left(\alpha_{1}-1\right)}<1$ and $\frac{\gamma_{1} \gamma_{2}}{\beta_{1} \beta_{2}}>\frac{\gamma_{1}\left(\alpha_{2}-1\right)}{\beta_{2}\left(\alpha_{1}-1\right)}$ are jointly true, then the only stable equilibrium is $E_{N}$, where only innovators survive;

IV. if $\frac{\gamma_{1}\left(\alpha_{2}-1\right)}{\beta_{2}\left(\alpha_{1}-1\right)}>1$ and $\frac{\gamma_{1} \gamma_{2}}{\beta_{1} \beta_{2}}<\frac{\gamma_{1}\left(\alpha_{2}-1\right)}{\beta_{2}\left(\alpha_{1}-1\right)}$ are jointly true, then the only stable equilibrium is $E_{M}$, where only imitators survive.

It is important to note that whenever an equilibrium is (locally) stable, its coordinates are not negative, so it is meaningful. In what follows, we will study the implications stemming from Proposition 1 for each of the two scenarios.

\subsection{First scenario (Scen-1): the prey-predator model}

It is useful to break down this scenario into two subcases. The first one in which $0<\alpha_{2}<1$ and the other one in which $\alpha_{2}>1$.

\section{First subcase}

With $\alpha_{2}$ less than unity it is immediate to prove that cases II. and IV. described in Proposition 1 are not feasible. In fact, we have that:

$$
\frac{\gamma_{1}\left(\alpha_{2}-1\right)}{\beta_{2}\left(\alpha_{1}-1\right)}<0
$$

Only the other two cases are feasible, so depending on the values of the parameters either the coexistence equilibrium is stable (case I.) or only innovators survive in the market (case III.). It is possible to identify a threshold value of $\beta_{1}$ (let us denote it as $\bar{\beta}_{1}$ ) such that:

- if $\beta_{1}<\bar{\beta}_{1}$, then $E^{*}$ is stable;

- if $\beta_{1}>\bar{\beta}_{1}$, then $E_{N}$ is stable;

- when $\beta_{1}=\bar{\beta}_{1}$ then $E^{*}=E_{N}$. In the dynamical systems theory this particular value of the parameter is called saddle-node bifurcation value. When a saddle-node bifurcation occurs two equilibria invert their stability properties, i.g. if for values of the parameter lower than the bifurcation one, an equilibrium is locally stable and the other is unstable, for values above the bifurcation value the opposite applies.

\footnotetext{
${ }^{6}$ Actually they were interested only in the parameters configurations corresponding to our second scenario. However, their results are more general and hold also for our first scenario.
} 
where

$$
\bar{\beta}_{1}=\frac{\gamma_{2}\left(\alpha_{1}-1\right)}{\alpha_{2}-1} .
$$

Figure 1a shows this situation in the $\left(\beta_{1}, \beta_{2}\right)$ parameters plane. This result implies that in order to guarantee the coexistence, low values of $\beta_{1}$ (below the threshold $\bar{\beta}_{1}$ ) are required. Higher values of $\alpha_{1}$ and $\gamma_{2}$ and/or lower values of $\alpha_{2}$ make the threshold $\bar{\beta}_{1}$ increase, favouring the convergence to $E^{*}$. By converse, if $\beta_{1}$ is higher than $\bar{\beta}_{1}$, then imitators are pushed out of the marker and only innovators survive. This case is easier to be achieved whenever the threshold $\bar{\beta}_{1}$ is low, and this occurs for low values of $\alpha_{1}$ and $\gamma_{2}$ and/or for high values of $\alpha_{2}$. Notice that the parameter $\beta_{2}$ plays no role in this subcase.

\section{Second subcase}

As far as the second subcase is concerned, i.e. $\alpha_{2}>1$, the situation definitely changes. Now, under this restriction it is easy to show that cases II. and III. described in Proposition 1 are not feasible. In fact:

$$
\frac{\gamma_{1} \gamma_{2}}{\beta_{1} \beta_{2}}<0 \text { and } \frac{\gamma_{1}\left(\alpha_{2}-1\right)}{\beta_{2}\left(\alpha_{1}-1\right)}>0 \quad \Rightarrow \quad \frac{\gamma_{1}\left(\alpha_{2}-1\right)}{\beta_{2}\left(\alpha_{1}-1\right)}>\frac{\gamma_{1} \gamma_{2}}{\beta_{1} \beta_{2}}
$$

So, either the coexistence equilibrium is stable (case I.) or innovators go out of the market and only imitators survive (case IV.).

Similarly to the previous subcase, we can identify a threshold value of $\beta_{2}$ (let us denote it as $\bar{\beta}_{2}$ ) separating these two possibilities:

$$
\begin{aligned}
& \text { if } \beta_{2}<\bar{\beta}_{2} \Longrightarrow E_{M} \text { is stable } \\
& \text { if } \beta_{2}>\bar{\beta}_{2} \Longrightarrow E^{*} \text { is stable }
\end{aligned}
$$

where

$$
\bar{\beta}_{2}=\frac{\gamma_{1}\left(\alpha_{2}-1\right)}{\alpha_{1}-1}
$$

Figure $1 \mathrm{~b}$ represents the situation in the $\left(\beta_{1}, \beta_{2}\right)$ parameters plane. Like for the previous subcase we can look deeply at the role played by the parameters. In order to guarantee a long-run coexistence of both kinds of firms, high values of $\beta_{2}$ (above the threshold $\bar{\beta}_{2}$ ) are required. This instance is easier to be achieved whenever $\bar{\beta}_{2}$ takes on low values and this occurs for relatively high values of $\alpha_{1}$ or sufficiently low values of $\gamma_{1}$ and/or $\alpha_{2}$. Otherwise, innovators are doomed to leave the market. Symmetrically, now $\beta_{1}$ does not play any role.

\section{FIGURE 1 HERE}

\subsection{Second Scenario (Scen-2): competition}

In the second scenario each of the two typologies of firms (innovators and imitators) inhibits the growth of the other. For this reason, this scenario can be paralleled to the two competing species analyzed by Leslie and Gower (1958). By varying the parameters values it is possible to verify all the four cases described in Proposition 1.

In particular, we can solve the four conditions of Proposition 1 with respect to $\beta_{1}$ or $\beta_{2}$ to obtain the following results:

$$
\begin{aligned}
& \beta_{1}>\bar{\beta}_{1} \text { and } \beta_{2}>\bar{\beta}_{2} \quad \Longrightarrow \quad E^{*} \text { is stable } \\
& \beta_{1}<\bar{\beta}_{1} \text { and } \beta_{2}<\bar{\beta}_{2} \quad \Longrightarrow \quad E_{N} \text { and } E_{M} \text { are both stable and coexist } \\
& \beta_{1}<\bar{\beta}_{1} \text { and } \beta_{2}>\bar{\beta}_{2} \Longrightarrow E_{N} \text { is stable and it is the only attractor } \\
& \beta_{1}>\bar{\beta}_{1} \text { and } \beta_{2}<\bar{\beta}_{2} \quad \Longrightarrow \quad E_{M} \text { is stable and it is the only attractor }
\end{aligned}
$$


where $\bar{\beta}_{1}$ and $\bar{\beta}_{2}$ are defined as in (33) and (36), respectively. The four cases can also be graphically identified in the $\left(\beta_{1}, \beta_{2}\right)$ parameters plane (Fig. 2$)$.

\section{FIGURE 2 HERE}

The main novelty of this scenario concerns the fact that case II of Proposition 1 is now feasible. We note that this is the only case in which the initial levels of innovators and imitators affect the final dynamic outcome. In the theory of dynamical systems, the set of initial conditions leading to an attractor are said to form the basin of attraction of the attractor. In our case we can numerically identify the two basins of attraction of the coexisting one-category equilibria and one example is provided in Figure 3.

\section{FIGURE 3 HERE}

The set of blue points corresponds to the initial levels of innovators and imitators that lead to $E_{M}$, in which only imitators survive. Instead, the red points characterize the initial conditions associated to the equilibrium with no imitators, $E_{N}$. The coexistence equilibrium $E^{*}$, even if locally unstable, has the property of being always located on the boundary separating the two basins of attraction ${ }^{7}$. As a consequence, the relative number of imitators and innovators in $E^{*}$ can be considered as a good proxy of the relative size of the two basins of attraction.

\section{Implications of our analysis}

The analytical and numerical analysis carried out in the previous Section provides us with the possibility to gain a better insight into the economic interpretation of system (28), especially with a particular focus on the coexistence equilibrium, which is the most interesting in our case as it practically captures the actual situation of any country.

In particular, as far as the prey-predator scenario is concerned (scen-1), in the first subcase, i.e. $0<\alpha_{2}<1$, we have claimed that higher values of $\alpha_{1}$ and $\gamma_{2}$ and/or lower values of $\alpha_{2}$ make the threshold $\bar{\beta}_{1}$ increase, favoring the convergence to $E^{*}$. By virtue of the relationships in (29) we know that the interval in which $\alpha_{2}$ lies implies $g<0$, that is, a negative imitators natural birth rate. In turn, for given $\alpha_{2}$, higher $\gamma_{2}$ implies higher imitators growth rate due to the interaction with innovators, $c$. Furthermore, higher $\alpha_{1}$ implies higher innovators birth rate, $r$. Therefore, a stable coexistence equilibrium is more likely the higher the innovators birth rate, the lower the imitators natural birth rate and the higher their birth rate stemming from hunting innovators. This threefold condition is consistent with a situation in which innovators grow at a substantial rate (high $r$ ), imitators are efficient in imitating innovators (high $c)$, but are a few $(g<0)$.

In the second subcase, i.e. $\alpha_{2}>1$, we have claimed that relatively high values of $\alpha_{1}$ or sufficiently low values of $\gamma_{1}$ and/or $\alpha_{2}$ make the threshold $\bar{\beta}_{2}$ decrease, making more likely convergence to $E^{*}$. By virtue of (29) we know that higher $\alpha_{1}$ implies higher innovators birth rate, $r$, low $\alpha_{2}$ implies low imitators birth rate (but still positive, given that $\alpha_{2}>1$ ) and eventually, for given $\alpha_{1}$ a low value of $\gamma_{1}$ implies a low innovators death rate induced by imitators (low $f$ ). Roughly speaking, this threefold condition shares with the previous one the high innovators birth rate and the low (but higher than the previous) imitators birth rate, but differently, it is consistent with a situation in which imitators are relatively weak,

\footnotetext{
${ }^{7}$ Technically speaking, the basins' boundary is the stable manifold of the unstable coexistence equilibrium.
} 
as they are not quite efficient in killing innovators (low $f$ ).

At this point the results may seem puzzling in that in the first subcase we have negative imitators birth rate versus a positive birth in the second subcase, but both lead to a stable coexistence equilibrium. The point is that in the first subcase, the negative birth rate is offset by efficiency in thriving with the chase (high $c$ ), while in the second subcase the positive birth rate is offset by scant efficiency in the killing rate (low $f$ ). This last remark highlights that innovation is the true engine of economic growth, imitation can help disseminating it, but must not be excessive. Most importantly, both subcases share the non trivial point that under certain circumstances $E^{*}$ is a feasible and stable configuration, namely innovators and imitators can coexist, without generating instability, here as a metaphor of an economic undesirable situation.

With reference to the second scenario (scen-2), characterized by competition between the species, a stable $E^{*}$ can occur when the inequalities $\beta_{1}>\bar{\beta}_{1}$ and $\beta_{2}>\bar{\beta}_{2}$ jointly hold. In order to provide an economic interpretation of this joint condition it is useful to comment on each inequality at a time, and then to make up the jigsaw puzzle. As regard to the condition on $\beta_{1}$, to occur it requires low values of $\alpha_{1}$ and $\gamma_{2}$ and/or high values of $\alpha_{2}$. In turn, this implies low $r$, low $c$ (which is negative) and high $g$. This combination of parameters captures the case in which few innovators are born, many imitators are born, but a great proportion of the latter are killed by the former (low $c$ ).

Whereas, $\beta_{2}>\bar{\beta}_{2}$ to occur requires relatively high values of $\alpha_{1}$ or sufficiently low values of $\gamma_{1}$ and/or $\alpha_{2}$. In turn, this implies high $r$, low $f$ and low $g$. This combination of parameters describes the case in which few imitators are born, many innovators are born and a little proportion of the latter are killed by the former (low $f$ ). The joint reading of the conditions on $\beta_{1}$ and $\beta_{2}$ is summarized in Table 2 .

Table 2: Synthesis of the conditions for a stable $E^{*}$ in a competitive environment

\begin{tabular}{|lll}
\hline \multicolumn{3}{|c|}{ condition on $\beta_{1}$} \\
$\left\{\begin{array}{lll}r & \text { low } & N \text { birth rate } \\
c & \text { low } & N \text { killing rate } \\
g & \text { high } & M \text { birth rate }\end{array}\right\}\left\{\begin{array}{lll}r & \text { high } & N \text { birth rate } \\
f & \text { low } & M \text { killing rate } \\
g & \text { low } & M \text { birth rate }\end{array}\right\}$ \\
\hline
\end{tabular}

The interpretation of Table 2 is quite easy. The birth rate of both species lies in an interval, being not too high and not too low, and both populations must not interact too aggressively, namely the killing rates must not be too high. The main message arising from this detailed economic interpretation is that a certain amount of competition between the two populations is desirable, but there must be a limit to the extent to which one population can hamper the others' activity. Put another way, a zero tolerance level towards imitation is neither feasible nor desirable, as suggested by Becker (1968) in a broader context. In this sense, the results are in line with that part of the literature postulating that the rate of innovation has an inverse- $U$ shape as a function of imitation. Although simple, this result captures the actual situation of most of the countries in which innovations coexist with imitations, both legal and illegal, thus showing the consistency of the evolutionary view of knowledge diffusion.

In addition, we can claim an even more general result which puts together all the instances, that is, a stable coexistence equilibrium can be achieved under any possible scenario: both prey-predator and competition. The same result does not apply to the other equilibria. As a very final remark, it is important to note that even if a stable coexistence equilibrium 
can exist, we do not know to what extent it can last. To the extent that a policy intervention alters the parameters entering the thresholds, $\beta_{1}$ and $\beta_{2}$, the conditions for $E^{*}$ may no longer apply. Roughly speaking, small changes in the parameters most likely will not alter the inequalities, while large changes are likely to make the inequalities no longer apply.

\section{Conclusions and further research}

This work accounts for a first move towards the microfoundation of the Lotka-Volterra view of the interplay between innovation and imitation. Starting from the model of Dixit and Pindyck (1994) set in a context of dynamic uncertainty and irreversible investment, we have derived the law of evolution of innovators and imitators in a competitive market. Thus, the model lends theoretical support to the evolutionary view which assumes that competitive relationships in the knowledge diffusion market can be analyzed by means of Lotka-Volterra codynamics. Put another way, this work brings out that there are microeconomic rationales to consistently assume a Lotka-Volterra evolution law. A thorough study of the equilibria shows that a stable coexistence equilibrium can be reached under both the prey-predator scenario and the pure competition. Gaining a better understanding of the dynamic interactions between firms who specialize in innovation and those who mainly imitate is an important topic and definitely needs additional investigation. As a further research, this work can be extended in several directions, both theoretical and empirical. Concerning the former, it would be worth investing in the endogenization of the entry thresholds, as well as some assumptions would deserve to be relaxed, such as the constant exit rate. As far as the empirical aspects are concerned, it would be worth attempting to estimate the parameters of the system for different markets and countries and simulate the impact of different policy interventions. A very similar exercise has been carried out by Lee et al. (2005) for two competing markets at the Korean stock market and by Kim et al. (2006) concerning the introduction of new mobile phones. Our future reseach efforts will focus on these issues.

Acknowledgment. Fabio Tramontana has been supported by the funds of the PRIN project "Local interactions and global dynamics in economics and finance:models and tools", MIUR, Italy. The opinions expressed by the authors are their only and do not necessarily reflect the positions of the Institutes.

\section{References}

[1] Aghion P., Bloom N., Blundell R., Griffith R., Howitt P., 2005. Competition and innovation: an inverted-U relationship. Quarterly Journal of Economics, 120, 701-728.

[2] Bharagava S.C., 1989. Generalized Lotka-Volterra equations and the mechanism of technological substitution. Technological Forecasting and Social Change, 35(4), 319326.

[3] Becker G.S., 1968. Crime and Punishment: an Economic Approach. Journal of Political Economy, 76(2), 160-217.

[4] Caballero R.J., Jaffe A.B., 1993. How high are the giants' shoulders: an empirical assessment of knowledge spillovers and creative destruction in a model of economic growth. In: Blanchard, O., Fischer, S. (Eds.), NBER Macroeconomics Annual. MIT Press, Cambridge, MA. 
[5] Castiaux A., 2007. Radical Innovation in established organizations: Being a Knowledge Predator. Journal of Engineering and Technology Management, 24, 36-52.

[6] Christensen C. M., 1997. The innovator's dilemma: When New Technologies Causes Great Firms to Fail. Harvard Business School Press.

[7] Christensen C. M., Raynir M.E., 2003. The Innovator's Solution: Creating Successful Growth. Harvard Business School Press.

[8] Cysne R.P., Turchick D., 2012. Intellectual property rights protection and endogenous economic growth revisited. Journal of Economic Dynamics $\&$ Control, 36, 851-861.

[9] Dixit A, Pindyck R., 1994. Investment under uncertainty. Princeton University Press.

[10] Furukawa Y., 2007. The protection of intellectual property rights and endogenous growth: is stronger always better?. Journal of Economic Dynamics $\&$ Control, 31, 3644-3670.

[11] Helpman E., 1993. Innovation, imitation, and intellectual property rights. Econometrica $61,1247-1280$.

[12] Judd K. L., 1985. The law of large numbers with a continuum of IID Random Variables. Journal of Economic Theory 35(1),19-25.

[13] Kim J., Lee D-J, Ahn J., 2006. A Dynamic competition analysis on the Korean Mobile Phone Market Using Competitive Diffusion Model. Computers and Industrial Engineering, 51(1), 174-218.

[14] Morris S., Pratt D. 2003. Analysis of the Lotka-Volterra competition equations as a technological substitution model. Technological Forecasting and Social Change, 70(2), 103-133.

[15] Lai E.L.C., 1998. International intellectual property rights protection and the rate of product innovation. Journal of Development Economics, 55(1), 133-153.

[16] Lee S-J., Lee D-J, Oh H-S., 2005. Technological Forecasting at the Korean Stock Market: A Dynamic competition Analysis Using Lotka-Volterra model. Technological Forecasting and Social Change, 72, 1044-1057.

[17] Leslie P.H., 1957. A stochastic model for studying the properties of certain biological system by numerical methods. Biometrika, 45, 16-31.

[18] Leslie P.H., Gower J.C., 1958. The properties of a stochastic model for two competing species. Biometrika, 45, 316-330.

[19] Lotka A.J., 1956. Elements of Mathematical Biology, Dover Pub. Inc. New York

[20] Odum E.P., 1971. Fundamentals of ecology, Third Edition. Saunders. London.

[21] Volterra V., 1926. Variazioni e fluttuazioni del numero d'individui in specie animali conviventi. Mem. Accad. Lincei Roma, 2, 31-113.

[22] Watanabe C. Kondo R., Nagamatsu A., 2003. Policy options for the Diffusion Orbit of Competitive Innovations - an Application of Lotka-Volterra Equations to Japan's Transition From Analog to Digital TV Broadcasting, Technovation, 23, 437-445. 
[23] Williamson, M., 1972. The Analysis of Biological Population. Edward Arnold. London. 


\section{CAPTIONS FOR FIGURES}

Figure 1: In (a) is represented the $\left(\beta_{1}, \beta_{2}\right)$ parameter plane corresponding to Scen-1 with $0<\alpha_{2}<1$. The vertical line $\beta_{1}=\overline{\beta_{1}}$ subdivides the plane into two regions. For values of $\beta_{1}$ lower than $\overline{\beta_{1}}$ the system converges towards the coexistence equilibrium, otherwise only innovators survive. In (b), with $\alpha_{2}>1$, the horizontal line $\beta_{2}=\overline{\beta_{2}}$ separates the region corresponding to a convergence to the coexistence equilibrium $\left(\beta_{2}>\overline{\beta_{2}}\right)$ from the region where only imitators survive $\left(\beta_{2}<\overline{\beta_{2}}\right)$.

Figure 2: The four regions that form the $\left(\beta_{1}, \beta_{2}\right)$ parameter plane in Scen-2.

Figure 3: Basins of attraction of the two coexistent equilibria $E_{N}$ (red basin) and $E_{M}$ (blue basin). The picture is obtained with this set of parameters: $\alpha_{1}=2, \alpha_{2}=1.5, \beta_{1}=1$, $\beta_{2}=0.25, \gamma_{1}=1$ and $\gamma_{2}=1.5$. 

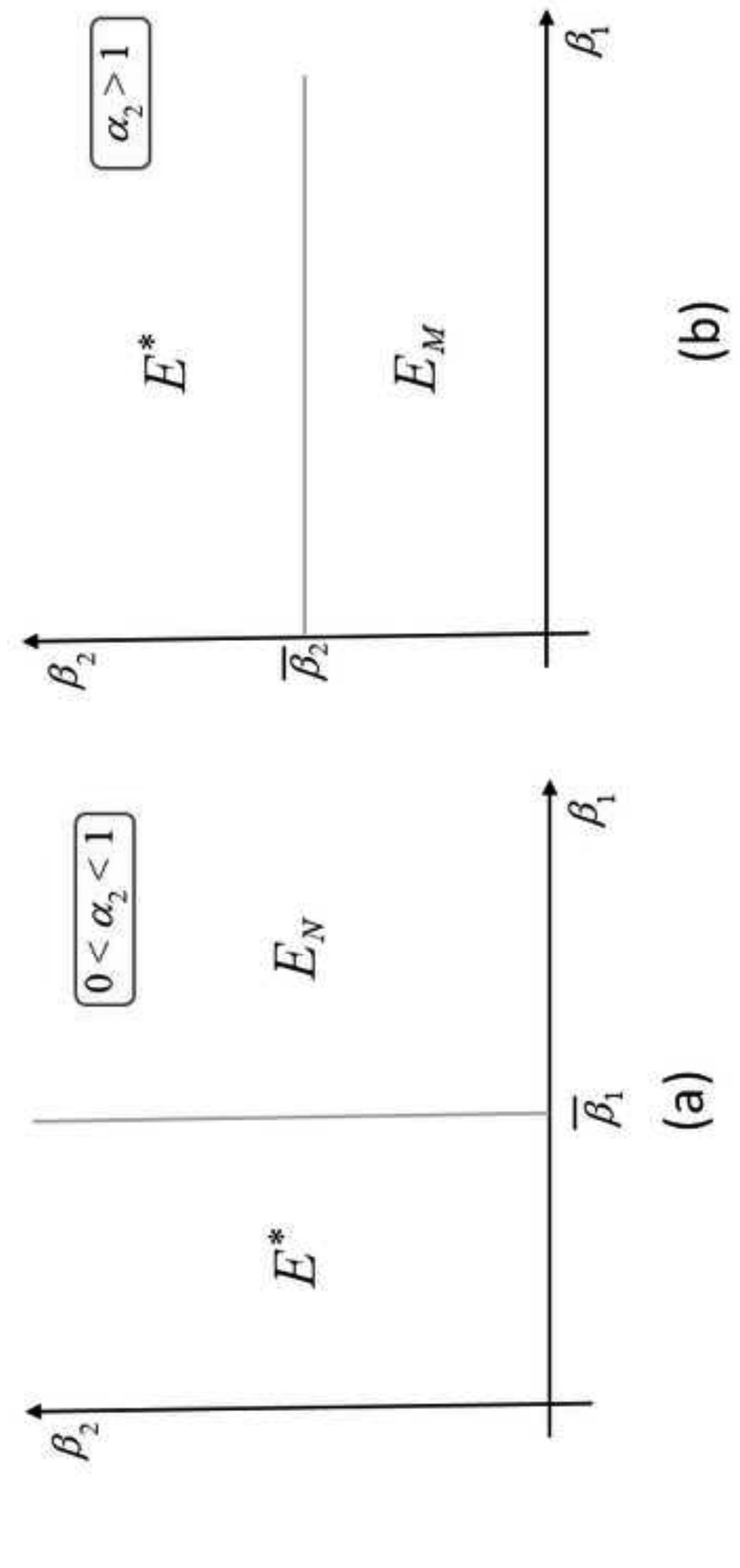


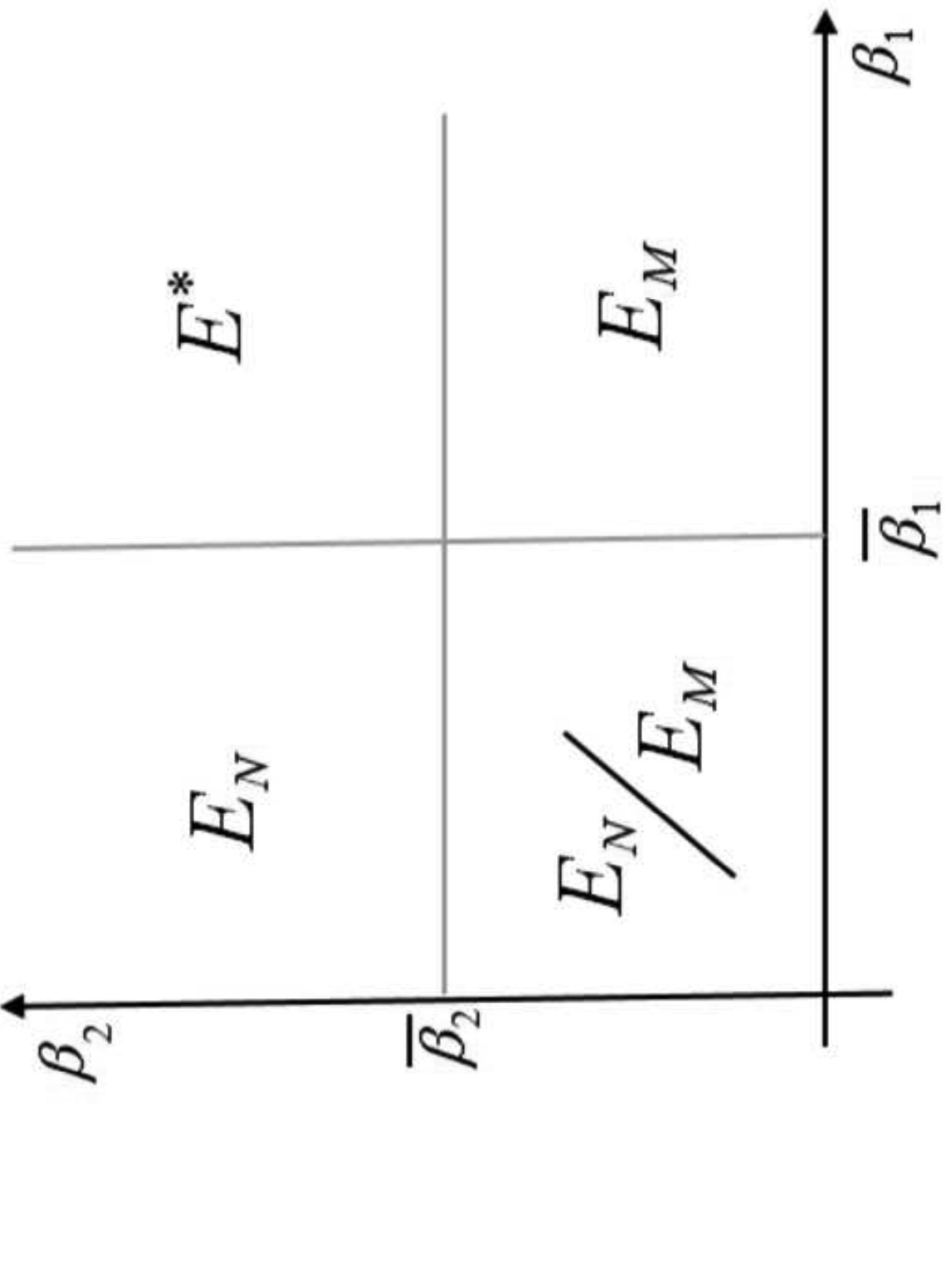




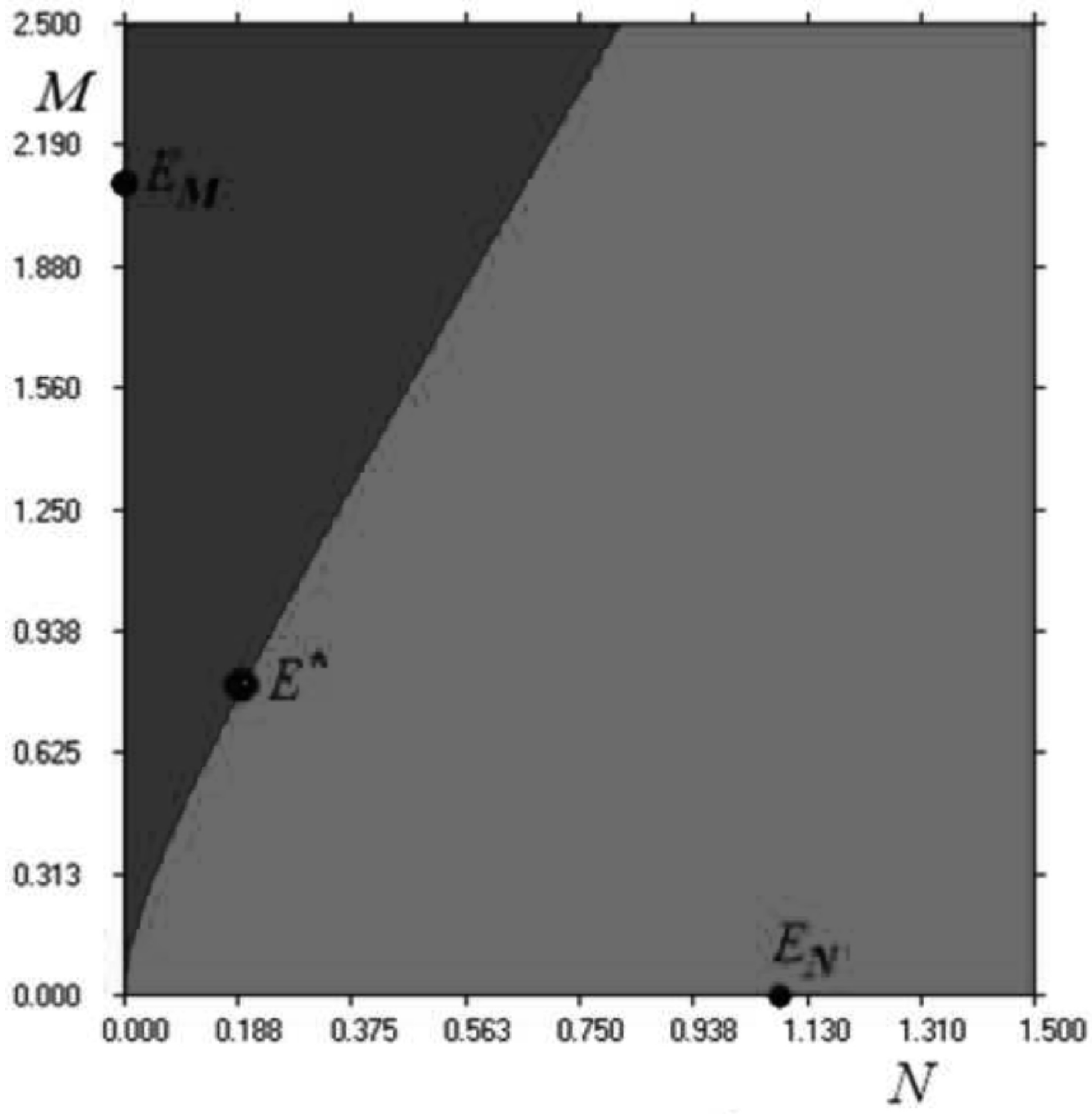

